\title{
Improvement of illumination-insensitive feathers for face recognition under complex illumination conditions
}

\author{
Liyun Zhuang ${ }^{1,2}$, Yepeng Guan*1,3 \\ 1. School of Communication and Information Engineering, Shanghai University \\ 2. Faculty of Electronic and Information Engineering, Huaiyin Institute of Technology \\ 3. Key Laboratory of Advanced Displays and System Application, Ministry of Education \\ Correspondence should be addressed to Yepeng Guan; shujpy@yeah.net
}

\begin{abstract}
Complex illumination condition is one of the most critical challenging problems for practical face recognition. In this paper, we propose a novel method to improve the illumination invariants for solving this challenge. Firstly, a new method based on the Lambert reflectance model is proposed to extract illumination invariant, which is less insensitive to complex illumination variations. Secondly, in order to repair the defects caused by process of illumination invariants extraction, Fast Mean Filter is utilized to smooth and remove noise. Lastly, for raising the richness of information in output image, a nonlinear normalization transformation is proposed. Compared with the state-of-the-arts, experimental results show that the proposed method can extract more robust illumination invariants. Apart from it, the richness of information in processed image is greater and superior performance in face recognition rate is superior.
\end{abstract}

Keywords: Improvement; illumination invariants; face recognition; complex illumination

\section{Introduction}

As an important research field of image processing and pattern recognition, face recognition has made remarkable progress in recent years. However, slight illumination changes can lead to great difference among the same target image, which increases the difficulty of image analysis and pattern recognition. Improvement of the illumination robustness for face recognition in complex light environment is a difficult problem in the field of artificial intelligence and computer vision [1,2].

At present, domestic and overseas researchers have proposed numerous illumination processing algorithms for face images under complex illumination conditions, and achieved good experimental results. They can be roughly divided into two categories. One approach deals with image processing modeling techniques, which are helpful to normalize faces with different lighting effects, such as logarithm transforms (LT) [3], gamma correction (GC) [4], block-based histogram equalization (BHE) [5], adaptive histogram equalization (AHE) [6], oriented local histogram equalization (OLHE) [7], the small-and large-scale (S\&L) features [8] and enhanced local texture feature [9]. LT and GC are of low computational complexity due to global techniques of image enhancement, but uneven illumination variation is too tricky even by using these global processing methods. On the other hand, AHE, BHE, AHE and OLHE also cannot achieve satisfactory performance on face recognition under complex illumination. The reason of this is that they tend to destroy overall distribution of essential image characteristics and enhancement of local texture feature tends to lose many image detail informations.

The other category directly or indirectly extracts illumination invariants, and it copes with illumination variation based on a common hypothesis that the reflectance changes quickly and the illumination varies slowly. This type of methods mainly consists of multiscale retinex (MSR) [10], DCT-based nor- 
malization technique (DCT) [11], self-quotient image (SQI) [12], logarithmic total variation (LTV) [13,14], gabor based methods [15], wavelet-based methods [16,17], contourlet-based methods [18], Bidimensional empirical mode decomposition (BEMD) [19], Gradient [20] and Weber [21]. MSR [10] combines several low-pass filters (i.e., Gaussian filters) for illumination estimation in a logarithm domain, which can cause serious halo effect. Gradient [20] is employed to reduce the halo effect in a certain degree based on an anisotropic filter. It is difficult for SQI [12] to keep sharp edges due to use of the weighted Gaussian filters. LTV $[13,14]$ improves SQI [12] based on logarithmic total variation. Furemore, illumination invariants extracted by wavelet-based [16,17] methods have obvious Gibbs phenomena and contourlet-based [18] methods tend to destory much useful information. BEMD [19] method has quite high computational expense. Gradient-based methods completely discard intensity information of facial reflectance. Cheng [22] proposes an illumination estimation algorithm to extract illumination invariants to meet the actual lighting situations and image acquisition model. In all, most of those methods based on the common hypothesis only extract high-frequency characteristics of illumination invariants (i.e., facial reflectance), neglect its low-frequency characteristic causing false edge phenomenon. On the other hand, most of them are sensitive to illumination variation. In addition, many image detail informations are easy to lose during the procedure of processing by most of those methods. Therefore, the enhancement of a facial texture feature is still unsatisfactory due to the superfluous information of facial skin.

In order to overcome the adverse effect of complex illumination variations on face recognition, an enhanced facial texture illumination normalization method is introduced in this paper. A novel illumination estimation algorithm is developed to extract illumination invariants, which can meet the actual lighting situations, image acquisition mode based on the analysis of the complex lighting conditions and the classical Lambertian reflectance mode. Fast mean filter is utilized to smooth processed image and remove noise. A nonlinear normalization transformation is developed to raise the richness of information in processed image. An overall flowchart for our proposed method is shown in Fig. 1. The main contributions of this paper are as follows: Firstly, the proposed method can more effectively extract illumination invariants, and it is less sensitive to complex illumination variation compared with other methods. Secondly, high-frequency and low-frequency characteristics are considered during the process of illumination invariants extraction, which can avoid loss of detail information. Thirdly, the richness of information is greater compared with other methods in the output image. Furthermore, experimental results indicate that the proposed method is a better approach compared with the state-of-the-arts.

This paper is organized as follows: Section 2 describes the proposed method. Section 3 shows experimental results and comparisons with state-of-the-art methods, and our concluding remarks are given in section 4. 


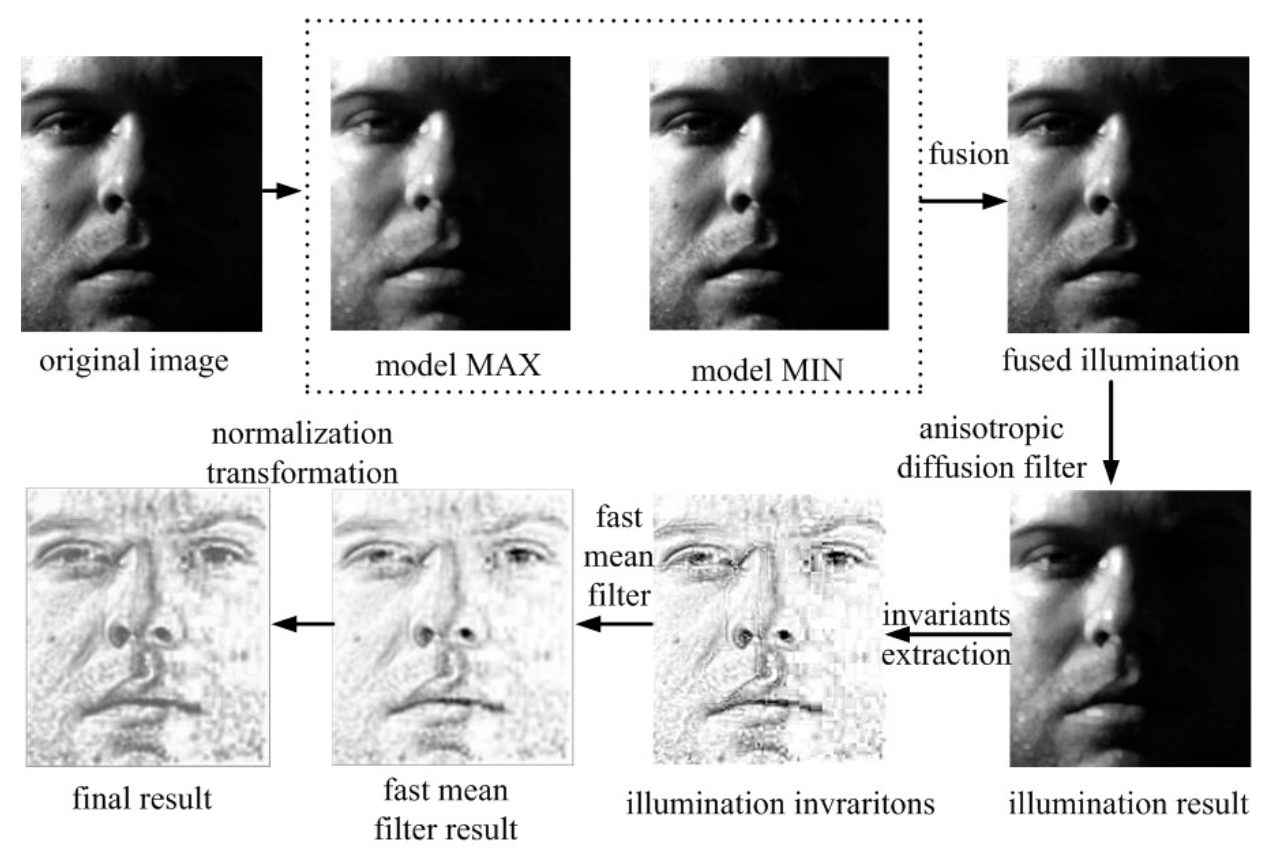

Fig. 1 The flowchart of proposed method

\section{Proposed Method}

\subsection{Image Acquisition Model}

Generally speaking, based on the classic Lambertian reflectance [23], image acquisition model regards an image as the multiplication of the illumination and the reflectance. In the Lambertian reflectance model, a facial image $I(x, y)$ can be formulated as

$$
I(x, y)=L(x, y) \times R(x, y)
$$

$L(\mathrm{x}, \mathrm{y})$ indicates illumination intensity and $R(x, y)$ denotes reflectance. Under different image acquisition environments, as the essential surface feature of human face, $R$ will keep unchanged while $L$ will change. In this case, facial reflectance $R$ can be looked upon as illumination invariants. From the above model defined by Eq. (1), we can know that if $R$ and $L$ are not known in advance, and it will be an illposed question to deduce $R$ and $L$ from an image. To deal with the problem, a common assumption is that $L$ varies slowly and $\mathrm{R}$ changes quickly. Under this assumption, an image is segmented into two frequency bands: low-frequency and high-frequency band, where $L$ and $R$ correspond to the low-frequency band and high-frequency, respectively. When illumination invariants are being extracted, only highfrequency characteristics of $R$ is considered while its low-frequency characteristics is ignored by many existing methods. However, in effect, a facial reflectance not only has high frequency characteristics, but also has low-frequency ones such as the reflectance in areas of eyes, eyebrow and skin. Both of them play important roles in face recognition. Therefore, assuming rapid changes of surface reflectance on human face is inappropriate, which easily causes extracted illumination invariants to lose important low-frequency information of facial reflectance. 


\subsection{Illumination Invariants}

It is usually assumed that illumination changes slowly by the conventional illumination estimation method. However, it causes failure to obtain good estimation result on images under complex illumination. Under ideal lighting conditions (parallel light without shielding, scattering or diffusing reflection phenomenon and so on), each image pixel usually has the similar illumination, and the reflectance value is between 0 and 1 . In this case, the maximum value of $I$ is closer to the illumination $L$ than other values of $I$. But in real environment the ideal illumination condition usually does not exist. For each image patch in the regions with slow illumination changes, the local maximum value of $I$ is a reasonable illumination estimation of its central pixel. The mode MAX is defined as

$$
L_{m}(x, y)=\max _{o_{i, j} \in \Omega}\left(I\left(o_{i, j}\right)\right)
$$

Where $\Omega$ indicates an image local window centered at $\operatorname{pixel}(x, y), O_{i, j}$ denotes a neighboring pixel in $\Omega$, and The functions $\max (\cdot)$ calculates the maximum value of a local window. Illumination estimation by local maximum pixel value is unsuitable for the pixels on the light shielding edge with quickly changed illumination. (3) is used to estimate illumination of pixels in the regions with quick illumination char(3) es. The mode MIN is described as

$$
\begin{gathered}
L_{s}(x, y)=\min _{o_{i, j} \in \Omega}\left(I\left(o_{i, j}\right)\right)+I(x, y) \\
I_{a}(x, y)=L_{m}(x, y)-I(x, y)
\end{gathered}
$$

The functions $\min (\cdot)$ calculates the minimum value of a local window. After calculating $L_{\mathrm{m}}$ and $L_{\mathrm{s}}$ of $I(x, y)$, illumination fusion is proposed to refine illumination estimation. In the fusion, we first distinguish between light shielding edges and other regions by image segmentation, and then define fused illumination estimation result $L_{m s}(x, y)$ of $I(x, y)$ by (5)

$$
\begin{gathered}
L_{m s}(x, y)=\left\{\begin{array}{cc}
L_{m}(x, y) & I_{g}(x, y) \geq \mathrm{t} \\
L_{s}(x, y) & I_{g}(x, y) \geq \mathrm{t}
\end{array}\right. \\
I_{g}(x, y)=I_{a}(x, y) / L_{m}(x, y) \\
t=\operatorname{mean}\left(I_{g}\right)+k \times\left(\max \left(I_{g}\right)-\operatorname{mean}\left(I_{g}\right)\right)
\end{gathered}
$$

Where mean $(\cdot)$ denotes the average value of an image and $k \in[0,1]$ is a parameter, which is set 0.6 according to [22]. For strong relationship among neighboring pixels of illuminations and better preservation of image edge information, anisotropic diffusion filter is proposed [24]. Equation of anisotropic diffusion is presented as

$$
L_{m s}^{t+1}(x, y)=L_{m s}^{t}(x, y)+\frac{\lambda}{\left|\eta_{(x, y)}\right|} \sum_{p \in \eta_{(x, y)}} g_{K}\left(\left|\nabla L_{((x, y), p)}\right|\right) \nabla L_{((x, y), p)}
$$

Where $(x, y)$ denotes the pixel position in the discrete 2-D grid; $t$ presents the iteration step; $K$ is the gradient threshold parameter; $\lambda$ determines the rate of diffusion and $\eta_{(x, y)}$ represents the spatial 4-pixel 
neighborhood of pixel $(x, y): \eta_{(x, y)}=\{N, S, E, W\}$, where $N, S, E$ and $W$ denote the North, South, East and West neighbors of pixel $(x, y)$, respectively. The symbol $\nabla$ which in the continuous form is used for the gradient operator now represents a scalar defined as the difference between neighboring pixels in each direction:

$$
\nabla L_{((x, y), p)}=L_{m s}^{t}(p)-L_{m s}^{t}(x, y) p \in \eta_{(x, y)}=\{N, S, E, W\}
$$

$g_{K}$ is the conductance function, and it is defined as

$$
g_{K}(x)=\exp \left[-\left(\frac{x}{K}\right)^{2}\right]
$$

The function (10) is chosen to satisfy $\lim _{x \rightarrow 0} g(x)=1$, so that the diffusion is maximal within uniform regions, and $\lim _{x \rightarrow \infty} g(x)=0$, so that the diffusion is stopped across edges. According to [24], $K$ $\lambda$ and $t$, respectively, are $15,0.15$ and 20 .

Based on the classic Lambertian reflectance model, illumination invariant at the coordinate of $(x, y)$ can be described as

$$
R(x, y)=\frac{I(x, y)}{L_{m s}(x, y)}
$$

Some illumination invariant results of different people under complex illumination conditions are shown in Fig. 2.
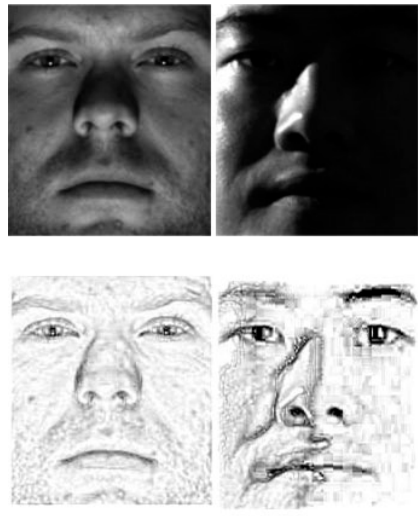

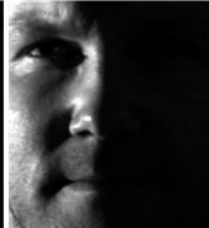

(a)
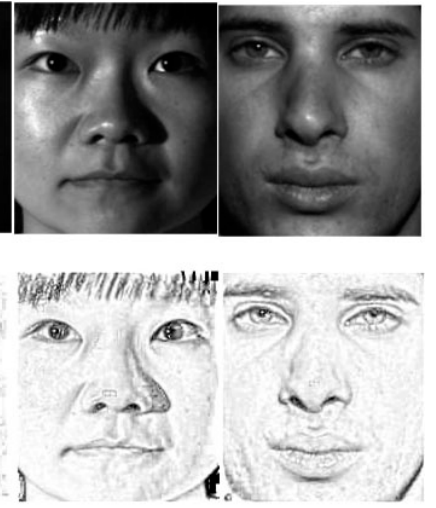

(b)

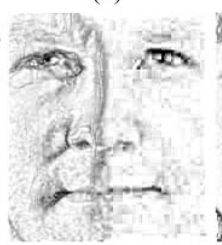

Fig.2 Illumination invariants (a) Original images (b) Results of illumination invariants

\subsection{Fast Mean Filter}

The invariants of illumination are extracted after illumination estimation, but unfortunately, the processed images suffer from loss of information, unsmooth texture and interference of noise. For further improvement of processed images, fast mean filter is used to smooth images and remove noise, which can repair the defects caused by process of illumination invariants extraction. 
The size of illumination invariant $R(x, y)$ is $P \times Q$, whose pixel greylevels are stored in a 2-D array, say, $\operatorname{data}[x][y]$ such that data[0][0] and data[P-1][Q-1] contains the first and last pixels, respectively. The algorithm is as follows

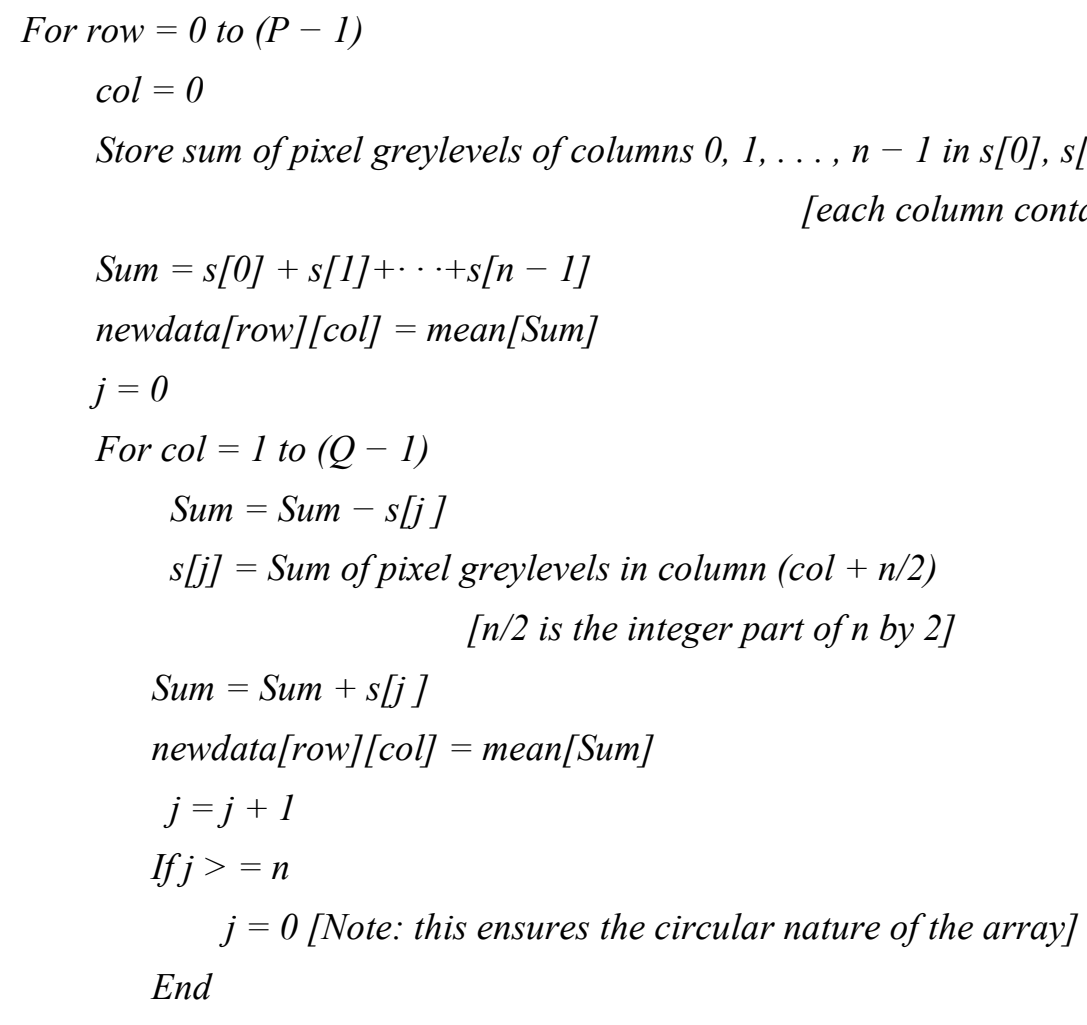

End

End

Compared with traditional gaussian low-pass filter, the filtering efficiency of fast mean filter is improved by about $50 \%$, and filter template size is empirically set $3 \times 3$ in regard to [25]. After fast mean filter processing, $R_{\text {FMF }}$ can be obtained. As shown in Fig. 3 , it is clear that the FMF results are smoother and have less noise compared with the results of illumination invariants.

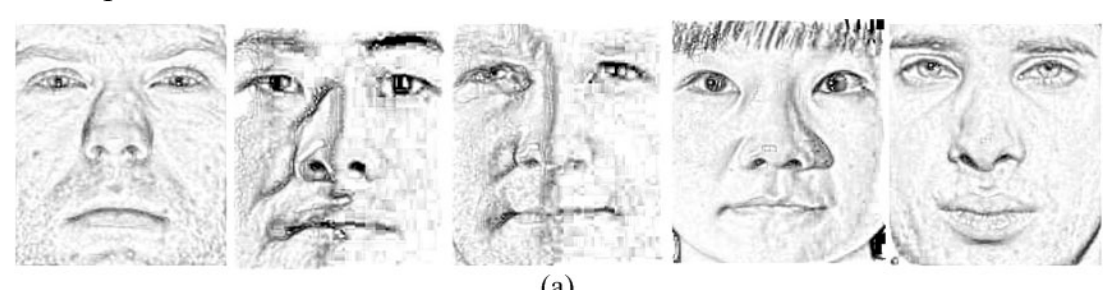

(a)

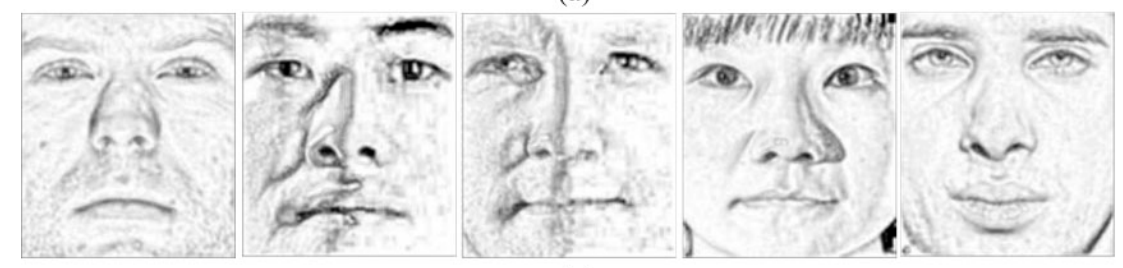

(b)

Fig. 3 (a) Results of illumination invariants used in Fig. 2 (b) FMF results of (a) 


\subsection{Normalization Transformation}

After smoothing the results of illumination invariants by utilizing fast mean filter, a normalization transformation is developed, which can raise the richness of information of the processed images. The transformation is defined as following:

$$
R_{\text {imp }}(x, y)=x^{\left(\log _{b}^{R_{F M F}(x, y)+a}+c\right)}
$$

Where $R_{F M F}: \Re^{N \times N} \rightarrow \Re^{N \times N}, x=\left(\max \left(l_{i j}\right)\right)^{1 /\left(\log _{b}^{l i j+a}+c\right)}, l_{i j}$ is the element of $R_{F M F}, a=\operatorname{mean}\left(R_{F M F}\right)$, $b=\operatorname{std}\left(R_{F M F}\right)$, and $c=\operatorname{mean}\left(R_{F M F}\right) / \operatorname{std}\left(R_{F M F}\right)$. Where the function mean $(\cdot)$ calculates the mean value of $R_{F M F}$, and std (') calculates the standard deviation value of $R_{F M F}$.

The transformation (12) would make the processed image have more details information and look more natural compared with the FMF results, as indicated in Fig. 4.

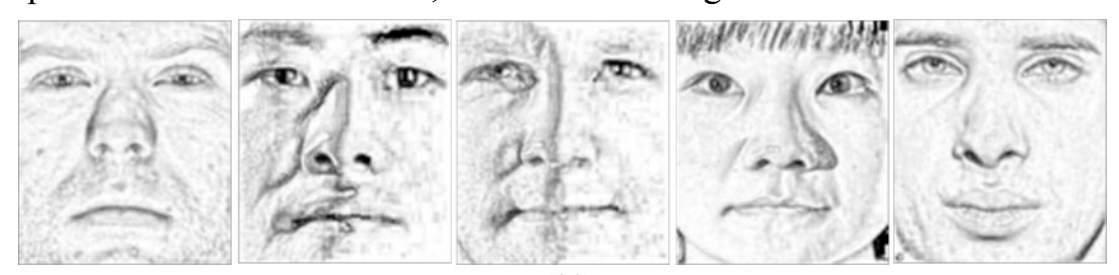

(a)

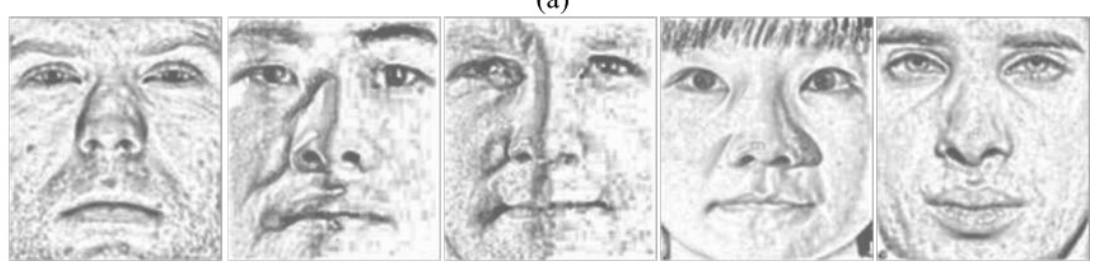

(b)

Fig. 4 (a) FMF results used in Fig. 3 (b) Results of normalization transformation

Fig. 5 shows the final results of different people. Original images selected from Yale B+ [27], CMUPIE [28] and CAS-PEAL-R1 [29] databases, which are under complex illumination conditions, as displayed in Fig. 5(a). The corresponding results by our method are given Fig. 5(b). The results show that the detail informations in final output images are greatly preserved by our method.

Entropy is a measure of the richness of information in the image, and the larger entropy value the image has, the higher the information contained in the output image.

The entropy for the whole image can be defined as

$$
D E=\sum_{l=0}^{L-1} e(l)=-\sum_{l=0}^{L-1} p(l) \log _{2} p(l)
$$

The entropy of the image can achieve maximum value only when $p(0)=p(1)=\ldots=p(L-1)=1 / L$ [26]. This is the scenario when the probability distribution of the image intensity values is uniform, which is the concept behind HE. Experiments on Yale B+ [27], CMU-PIE [28], CAS-PEAL-R1 [29] and ORL [30] databases show that the images processed by the proposed method can get the biggest DE values, as indicated in Table 1. 


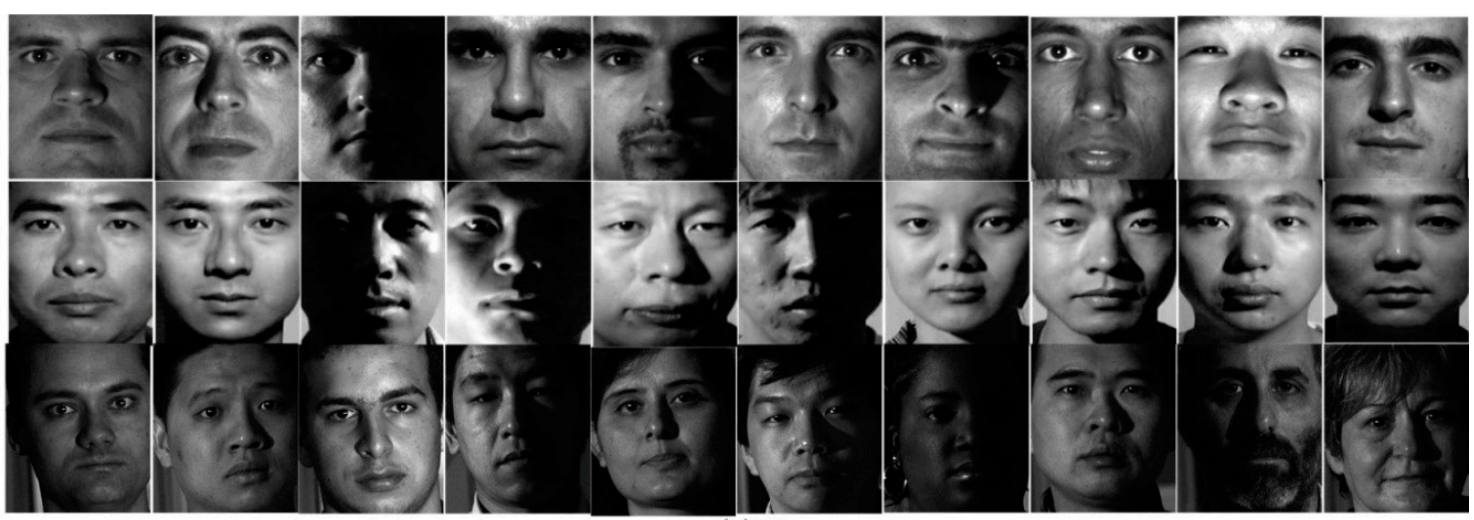

(a)

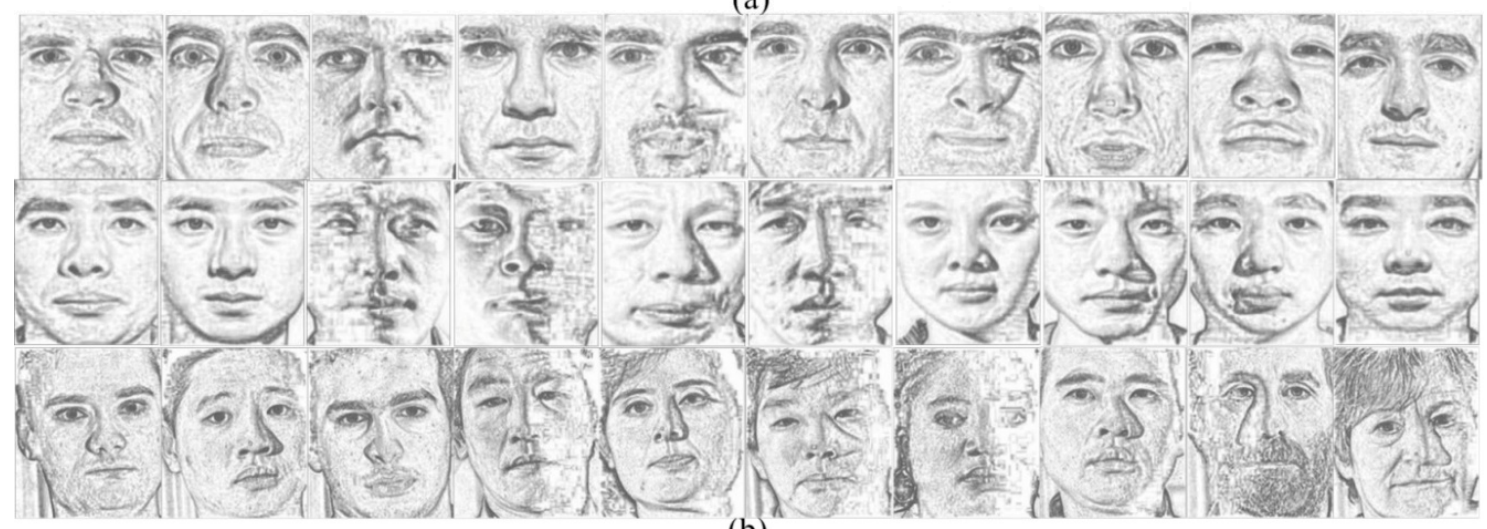

(b)

Fig. 5 (a) Original images (b) Final results of illumination invariants

Table 1. Entropy value comparisons in different methods

\begin{tabular}{lcccccc}
\hline Databases & \multicolumn{9}{c}{ Entropy value of different methods } & & \\
& MSR [10] & DCT[11] & Gradient[20] & Weber[21] & Cheng[22] & Proposed \\
\hline Yale B & 6.69 & 6.65 & 6.62 & 6.18 & 6.47 & $\mathbf{6 . 8 5}$ \\
CMU-PIE & 6.65 & 6.43 & 6.67 & 5.81 & 6.53 & $\mathbf{6 . 8 6}$ \\
CAS-PEAL-R1 & 6.51 & 6.47 & 6.56 & 6.23 & 6.31 & $\mathbf{6 . 7 1}$ \\
ORL & 6.75 & 6.72 & 6.79 & 6.89 & 6.55 & $\mathbf{6 . 9 8}$ \\
\hline
\end{tabular}

\section{Experimental Results}

In this section, Yale B+ [27,28], CMU-PIE [29], CAS-PEAL-R1 [30] and ORL [31] databases are selected to evaluate the performance of the method introduced in this paper. Face recognition on those databases are still a challenging task owing to their complex lighting conditions. Yale B+ consists of 38 subjects under 64 lighting conditions. The CMU-PIE includes 68 subjects under 21 illumination conditions with a little hair and background in the face images. The illumination of CAS-PEAL-R1 face database is different from Yale $\mathrm{B}+$, and the number of illumination conditions is not the same for each subject. Besides, there exists illumination interference in this database. Moreover, it is more difficult for face recognition because of the difference of poses and scale changes for the same person. The ORL face database contains 400 face images of 40 human subjects with varying lighting, facial expressions 
and facial details (glasses / no glasses). And all images are taken under a dark homogeneous background.

All selected images are resized to $64 \times 56$. According to the serial number order, one image of each subject is used as training samples, other images are used for testing, and the final result is calculated by the average value. PCA [32], KFA [33] and LDA [34] are used for testing in the same conditions, because they are highly sensitive to illumination variation. MSR [10], DCT [11], Gradient [20], Weber [21] and Cheng [22], as the state-of-the-art methods, are selected to implement performance comparison. The opening INface toolbox v2.1 [23] is utilized to achieve MSR [10], DCT [11], Gradient [20] and Weberface [21] with their recommended parameters by authors.

\subsection{Experiments on Yale $B+$ database}

In our experiments, we choose the frontal images under varying lighting conditions. The statistical results of recognition rates of PCA [32], KFA [33] and LDA [34] based on all the methods on Yale B+ database are shown in Fig. 4.

It can be seen from Fig. 6 that our introduced method performs the best in comparison with all other approaches, because it can more effectively preserve the image detail information and improve richness of illumination invariant. The recognition rates of PCA, KFA and LDA based on our method are $95.62 \%, 97.78 \%$ and $98.72 \%$, respectively. Specifically speaking, the recognition rate of performing the other approaches: MSR [10], DCT [11], Gradient [20], Weber [21] and Cheng [22] are discriminatively lower than performing our method on the Yale $\mathrm{B}+$ dataset containing with full illumination variation.

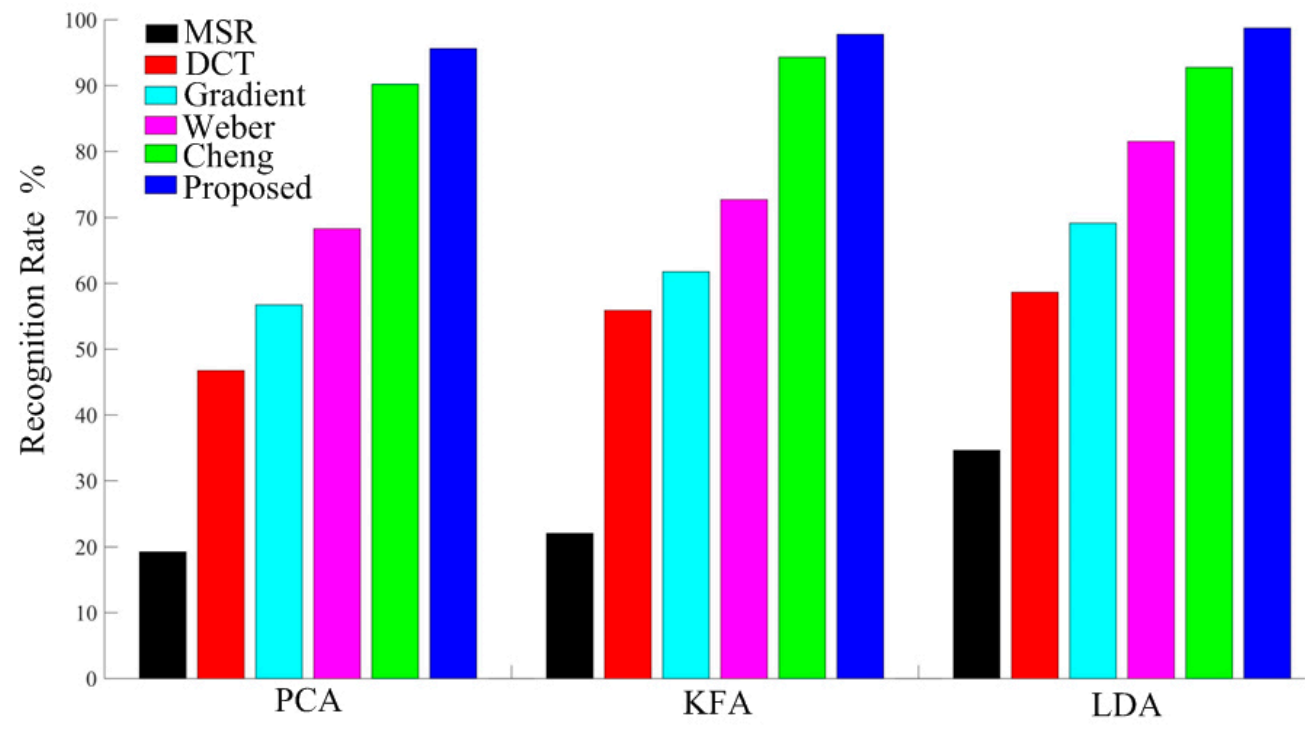

Fig. 6 Comparison of recognition rate on Yale $\mathrm{B}+$ database

Fig. 7(a) shows some original images of subjects from Yale $\mathrm{B}+$ dataset, and the corresponding results of different methods are indicated in Fig. 7(b) - Fig. 7(g). We can see that the original images are under complex illumination conditions. It is clear that the proposed method can more effectively retain facial 
detail informations and eliminate the influence of illumination change on digital images under complex illumination conditions compared with other methods.

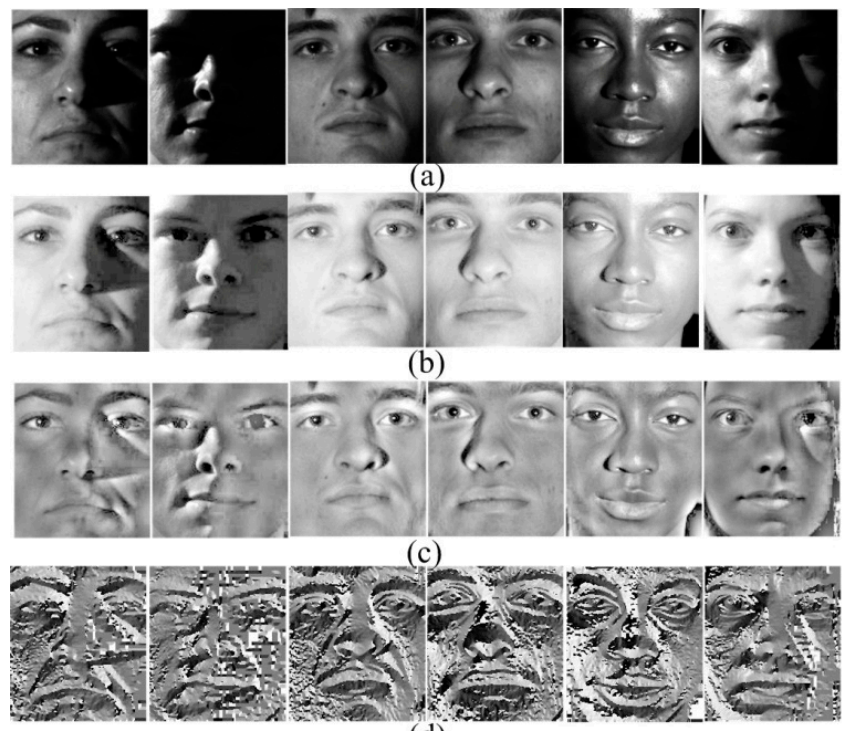

(d)

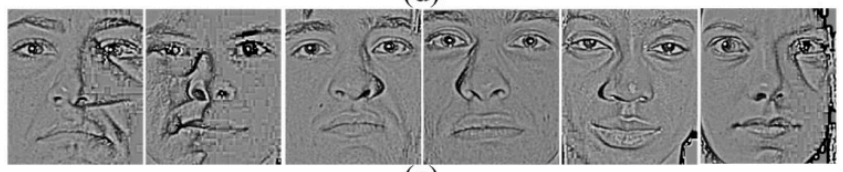

(e)

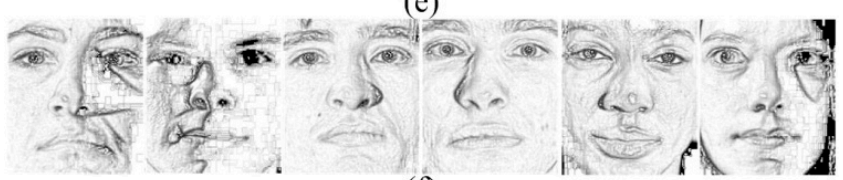

(f)

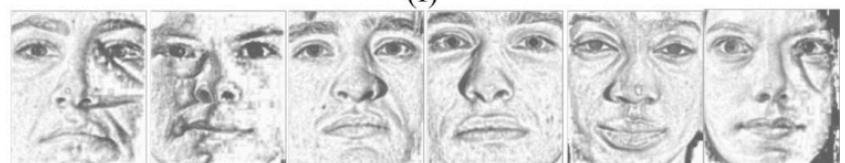

(g)

Fig. 7 The corresponding results of different methods on the Yale B+: a original images, b MSR [10], c DCT [11], d Gradient [20], e Weber [21], f Cheng [22], g Proposed

\subsection{Experiments on CMU-PIE database}

In this section, we choose the frontal images under varying lighting conditions from CMU-PIE database. The statistical results of recognition rates of PCA [32], KFA [33] and LDA [34] on CMU-PIE database are displayed in Fig. 8.

As indicated in Fig. 8, most of the methods can obtain high recognition. The recognition rate of performing our method is better compared with the other state-of-arts. The recognition rates of KPA based on the mentioned methods are bigger than $90 \%$ except MSR, and the proposed method can get the highest recognition rate (99.05\%). Additionally, the method introduced by us can also get the highest recognition rate by LDA. 
Fig. 9 shows some complex illumination images of subjects from CMU-PIE database and the corresponding results of different methods. Compared with some state-of-the-arts, it is clear that our approach performs best on retaining facial details and eliminating the influence of illumination change on digital images retaining facial details, which are displayed in Fig. 9(b) to Fig. 9(g).

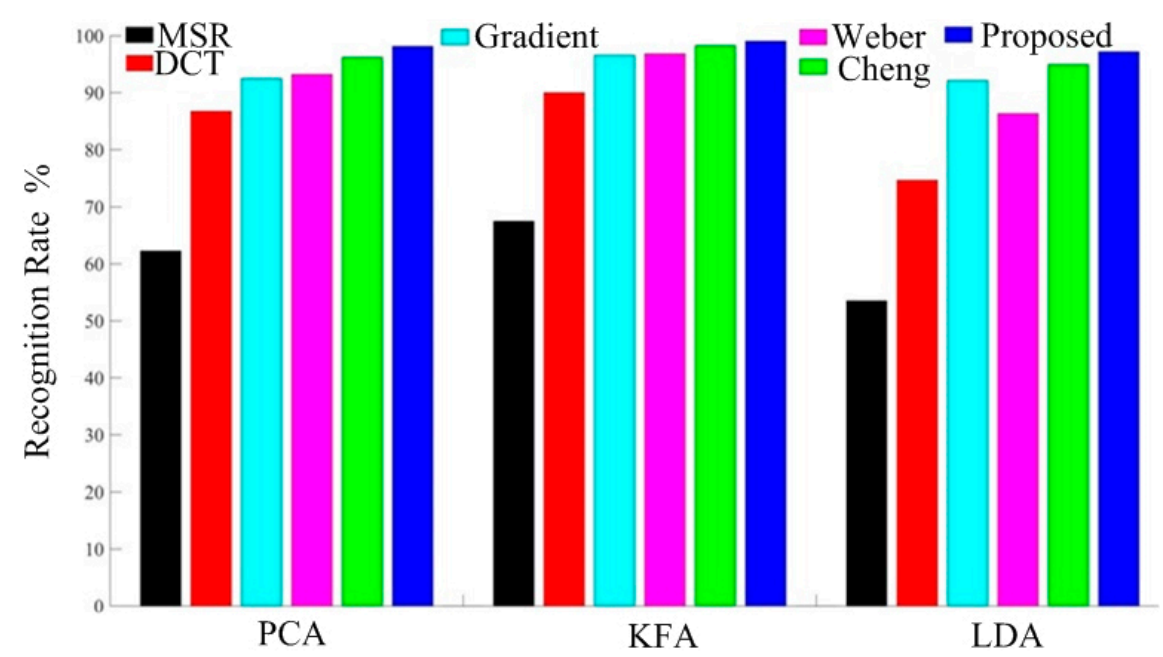

Fig. 8 Comparison of recognition rate on CMU-PIE database

\subsection{Experiments on CSA-PEAL-R1 database}

In order to build a fair comparison among the investigated approaches, 233 subjects under 10 different illuminations are chosen from CAS-PEAL-R1 database. Just like experiments on Yale $\mathrm{B}+$ and CMU-PIE databases, one illumination of each subject is selected randomly as a training sample, and the others are taken as a testing set for face recognition, the final result is calculated by the average value.

The recognition results of PCA [32], KFA [33] and LDA [34] are shown in Fig. 10. It can be known from Fig. 10 that the overall recognition rates by all the method is declined sharply. One reason is that the difference of poses and scale changes for the same person in CSA-PEAL-R1 database. One can find that our proposed approach performs the best by comparison with all other methods.

Some different lighting images of subjects and the corresponding results of different methods are indicated in Fig. 11. From Fig. 11(b) to Fig. 11(g), we can know that our method can more effectively retain facial details and eliminate the influence of illumination change on digital images, which are under complex illumination conditions. And the final illumination invariants processed by our method look more natural and have more detail information.

\subsection{Experiments on ORL database}

We use 400 face images of 40 human subjects in our experiments on ORL database. As indicated in Fig. 12, the results of recognition rates of PCA [32], KFA [33] and LDA [34] on ORL database are displayed. 


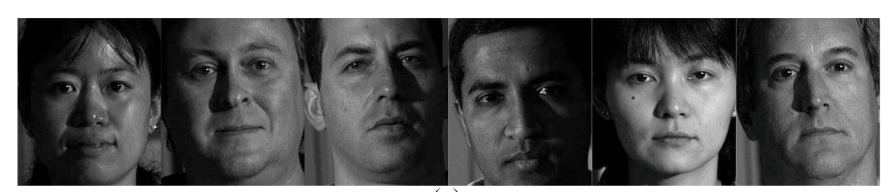

(a)

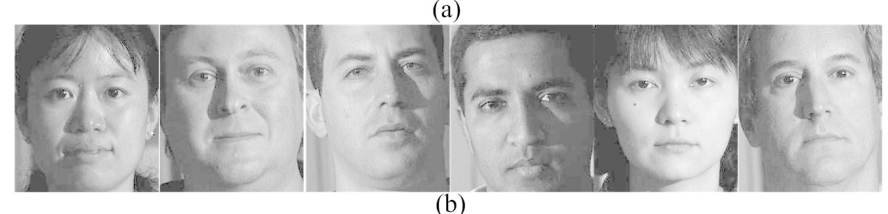

(b)

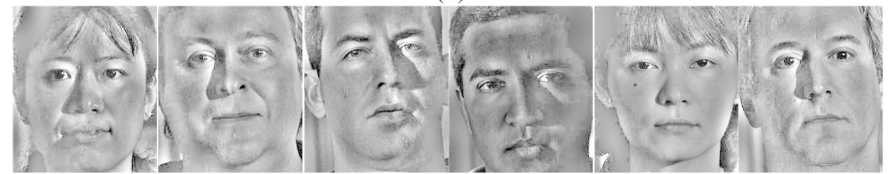

(c)
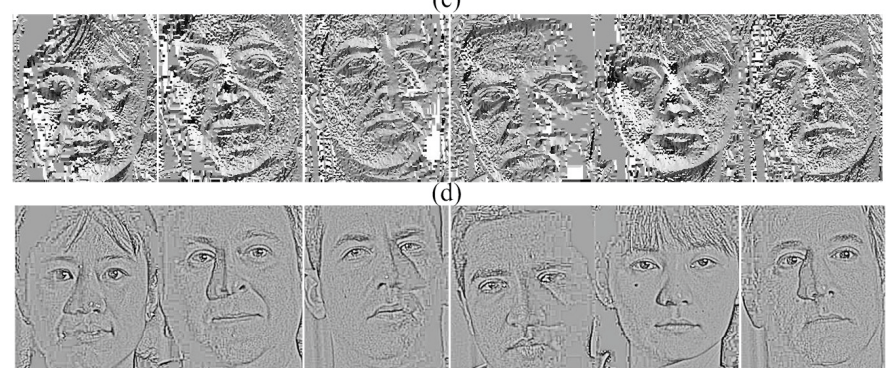

(e)

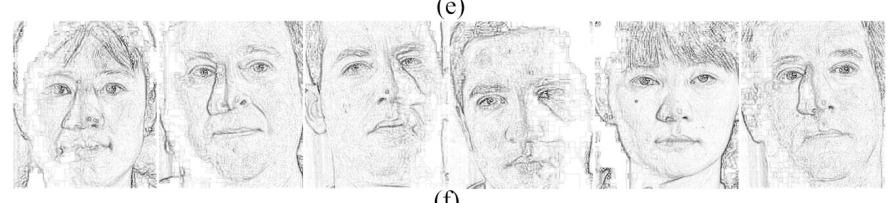

(f)

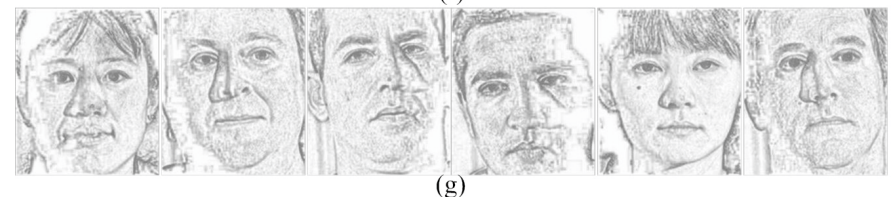

(g)

Fig. 9 The corresponding results of different methods on the CMU-PIE: a original images, b MSR [10], c DCT [11], d Gradient [20], e Weber [21], f Cheng [22], g Proposed

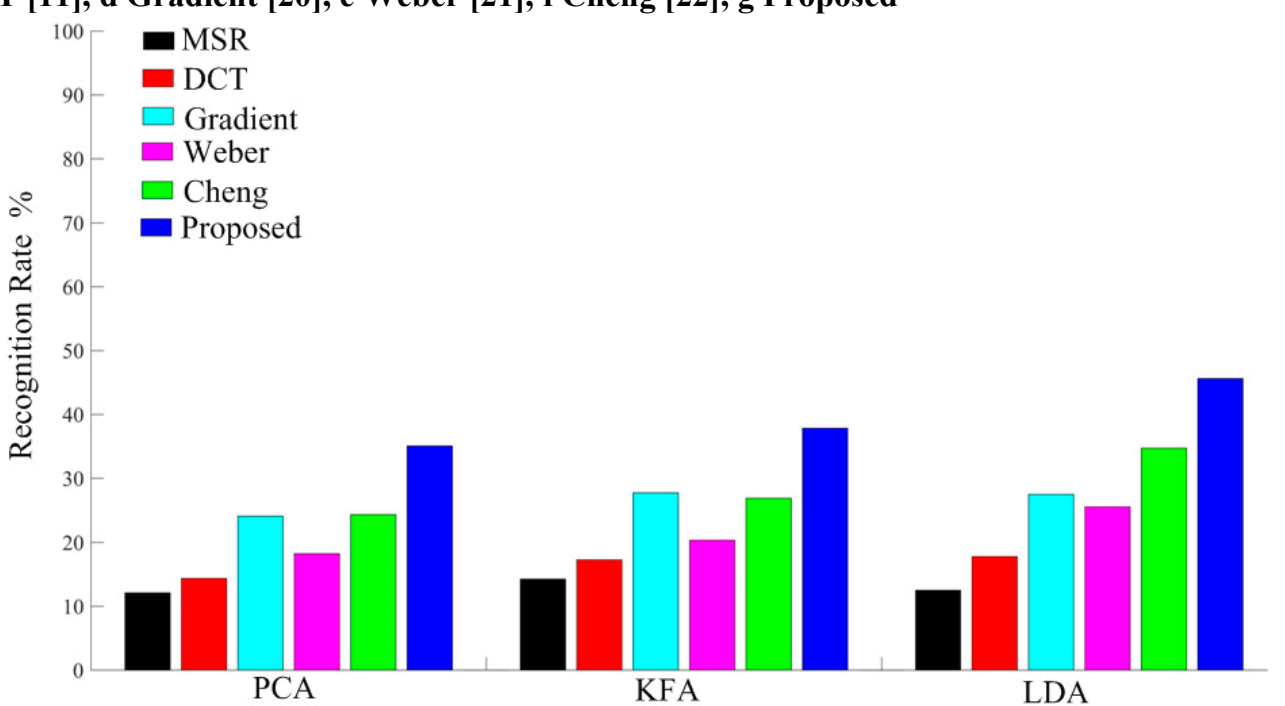

Fig. 10 Comparison of recognition rate on CSA-PEAL-R1 database 
Compared with other methods, we can know that our proposed approach performs the best, as shown in Fig. 12. The recognition rates of PCA based on our method can reach $50.31 \%$, and recognition rates of the others are lower than 50\%. Specifically speaking, the recognition rates of KFA and LDA based on our method are also perform best in comparison with the other approaches, which are $63.27 \%$ and $66.87 \%$, respectively.

Some images of subjects from ORL dataset and the corresponding results of different methods are indicated in Fig. 13. As shown in Fig. 13(a), we can see that the original images are under complex conditions, such as varying lighting, facial expressions and facial details (glasses / no glasses). The proposed methods can surpass the other methods in retaining facial details and eliminating the influence of illumination change on digital images under complex conditions, in Fig. 13(g),

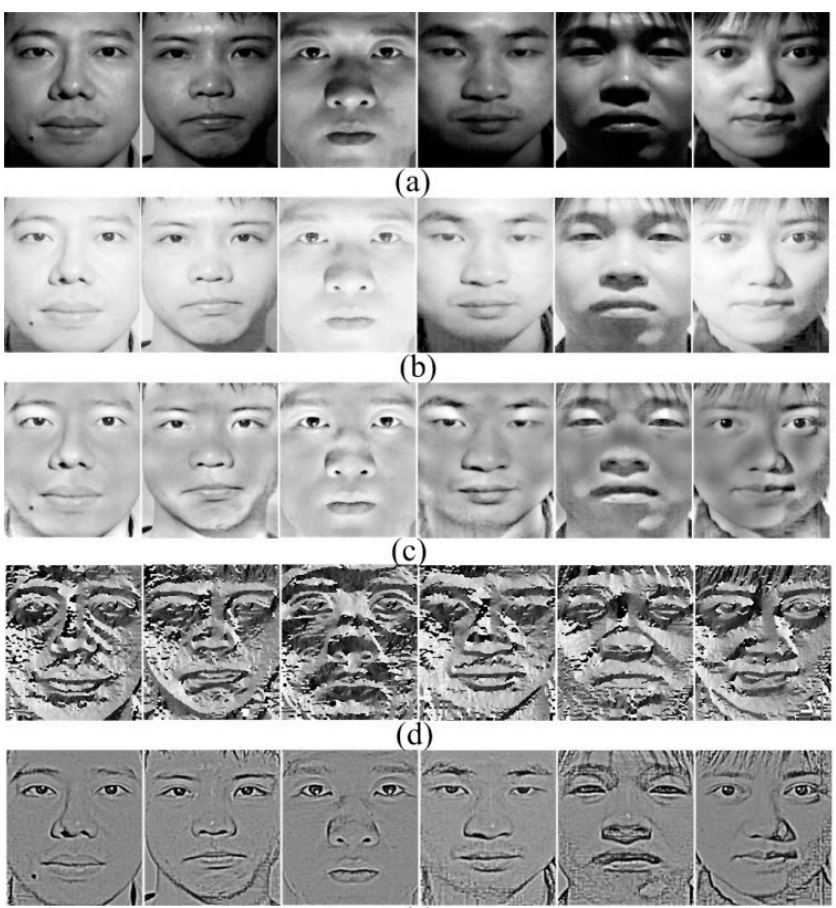

(e)

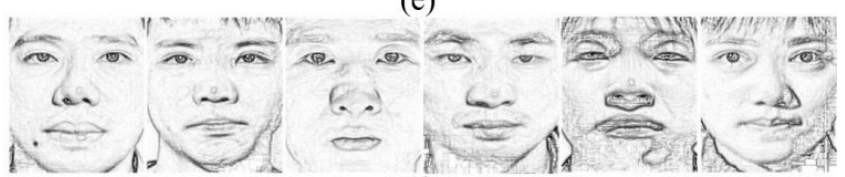

(f)

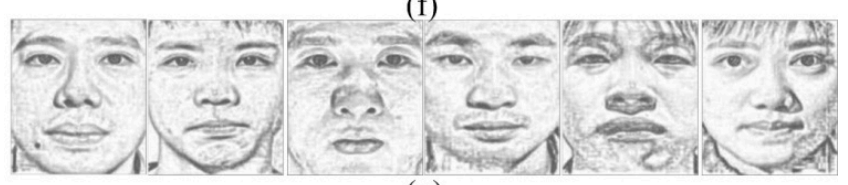

(g)

Fig. 11 The corresponding results of different methods on the CSA-PEAL-R1: a original images, b MSR [10], c DCT [11], d Gradient [20], e Weber [21], f Cheng [22], g Proposed 


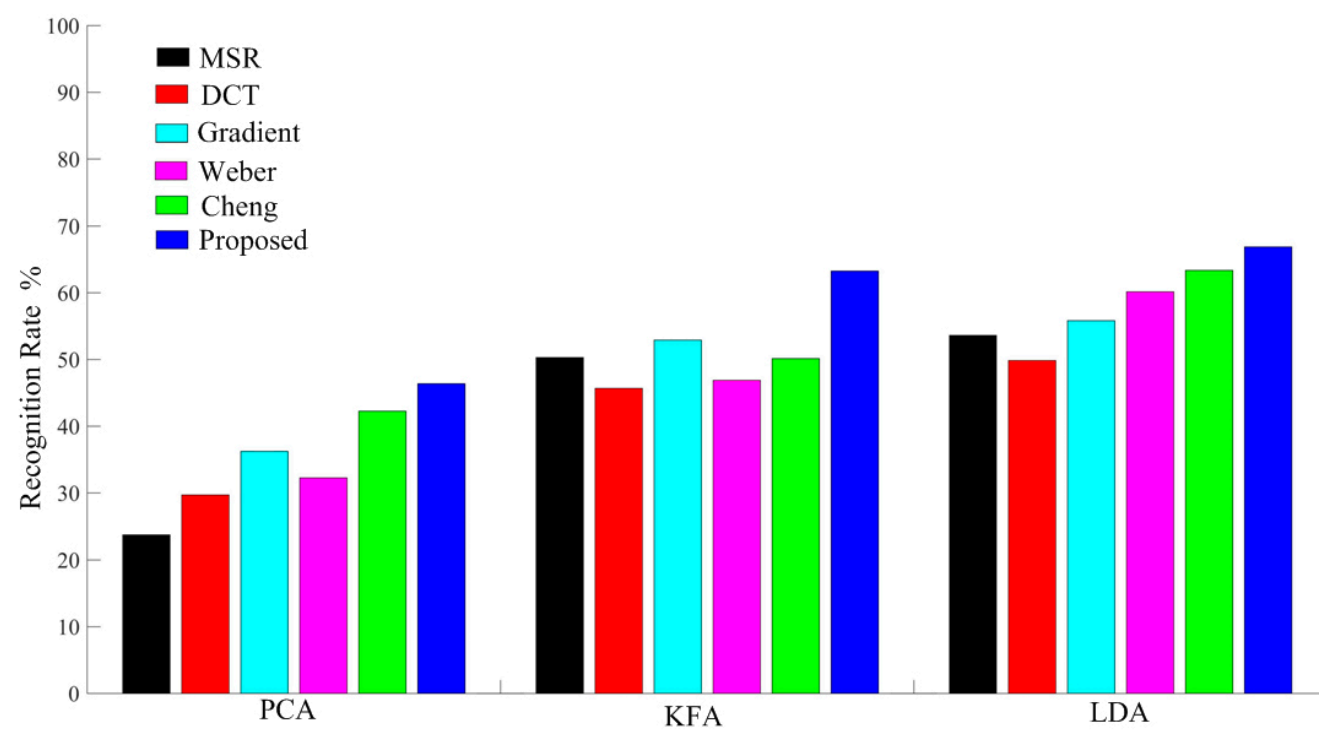

Fig. 12 Comparison of recognition rate on ORL database

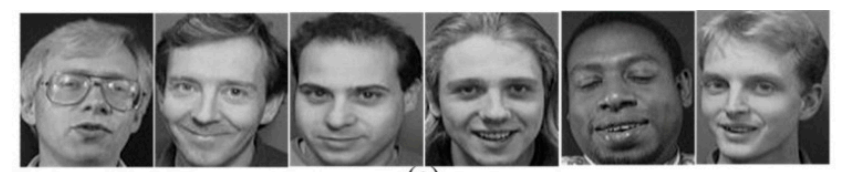

(a)

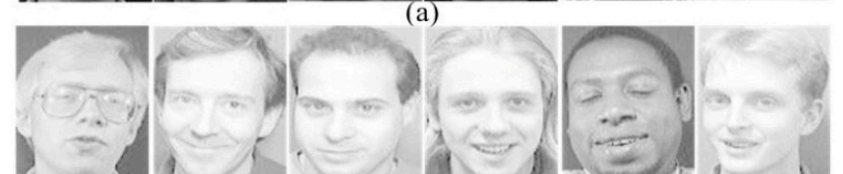

(b)

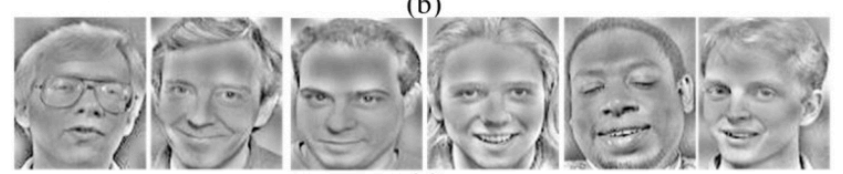

(c)

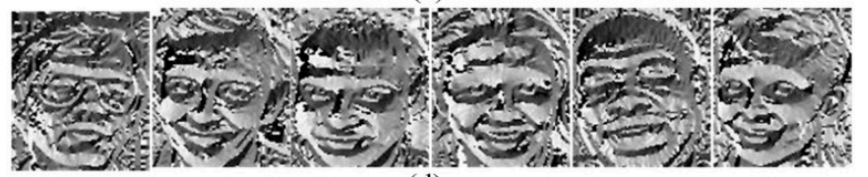

(d)

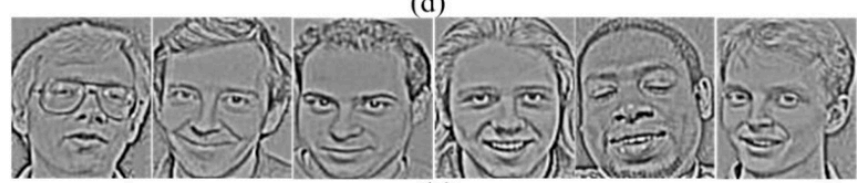

(e)

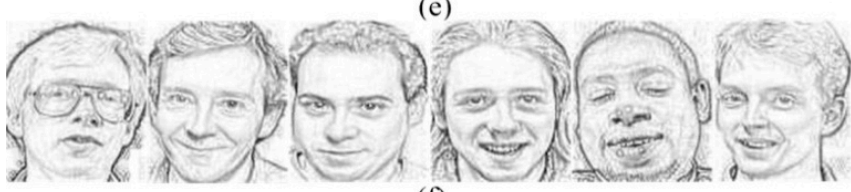

(f)

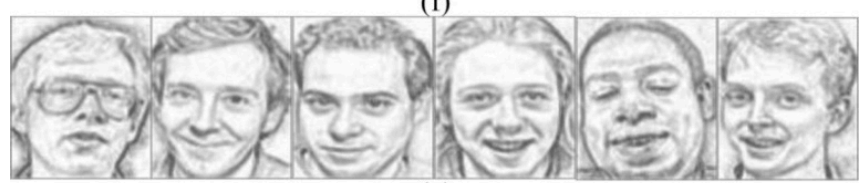

(g)

Fig. 13 The corresponding results of different methods on the ORL: a original images, b MSR [10], c DCT [11], d Gradient [20], e Weber [21], f Cheng [22], g Proposed 
To sum up, as indicated in Fig. 6, 8, 10, and 12. The MSR method has the lowest recognition rates on Yale B+, CMU-PIE, CAS-PEAL-R1 databases. But on the ORL database, the recognition rates of MSR are higher than DCT by KFA and LDA. DCT makes unsatisfactory performances in the four public databases, but in most cases, it performs better than MSR. The performance of Gradient is better than that of MSR and DCT on all the tested databases, however, the performance is still not ideal. Weber can obtain higher recognition rates than MSR, DCT and Gradient on Yale B+, and it is mostly lower than Gradient on the other three databases. The Cheng method ranks as the second in the four databases except on ORL by KFA. Through the results of the comparison mentioned above, it is clear that our method can obtain the best performance on the databases used in this paper.

\section{Conclusions}

An effective method is used to solve the critical issue of complex illumination variation for face recognition in this paper. The proposed method is less insensitive to complex illumination variation and repairs the defects caused by process of illumination invariants extraction by using fast mean filter. Improvement of illumination invariant by our method can make the output image get more detail information than other methods, thus a greater richness of information and higher recognition rate under complex illumination conditions can be obtained by the proposed method. Experimental results have shown that the proposed method has superior performance in face recognition by comparing with some state-of-the-arts.

\section{Availability of data and material}

All data are fully available without restriction.

\section{Competing interests}

The authors declare that they have no competing financial interests.

\section{Funding}

This work was supported by the National Natural Science Foundation of China under Grants 11176016 and 60872117.

\section{Authors' contributions}

LYZ implemented the core algorithm and drafted the manuscript. YPG reviewed and edited the manuscript. All authors discussed the results and implications, commented on the manuscript at all stages, and approved the final version.

\section{Acknowledgements}

Not applicable. 


\section{Authors' information}

Liyun Zhuang was born in Linyi, Shandong Province, China. He is now a Ph.D. Candidate of School of Communication and Information Engineering in Shanghai University, China. His major research interests include computer vision, pattern recognition.

Yepeng Guan was born in Xiaogan, Hubei Province, China, in 1967. He received the B.S. and M.S. degrees in physical geography from the Central South University, Changsha, China, in 1990, 1996, respectively, and the Ph.D. degree in geodetection and information technology from the Central South University, Changsha, China, in 2000. Since 2007, he has been a Professor with School of Communication and Information Engineering, Shanghai University.

\section{References}

[1]Phillips P J, Scruggs W T, O'Toole A J, et al. FRVT 2006 and ICE 2006 large-scale experimental results. IEEE transactions on pattern analysis and machine intelligence, 2010, 32(5): 831-846.

[2] Vageeswaran P, Mitra K, Chellappa R. Blur and illumination robust face recognition via set-theoretic characterization. IEEE transactions on image processing, 2013, 22(4): 1362-1372.

[3] Savvides M, Kumar B V K. Illumination normalization using logarithm transforms for face authentication. IEEE International Conference on Audio-and Video-Based Biometric Person Authentication, 2003: 1055-1055.

[4] Shan S, Gao W, Cao B, et al. Illumination normalization for robust face recognition against varying lighting conditions IEEE International Workshop on Analysis and Modeling of Faces and Gestures, 2003: 157164.

[5] Xie X, Lam K M. Face recognition under varying illumination based on a 2D face shape model. Pattern Recognition, 2005, 38(2): 221-230.

[6] Stark J A. Adaptive image contrast enhancement using generalizations of histogram equalization. IEEE Transactions on image processing, 2000, 9(5): 889-896.

[7] Lee P H, Wu S W, Hung Y P. Illumination compensation using oriented local histogram equalization and its application to face recognition. IEEE Transactions on Image processing, 2012, 21(9): 4280-4289.

[8] Xie X, Zheng W S, Lai J, et al. Normalization of face illumination based on large-and small-scale features. IEEE Transactions on Image Processing, 2011, 20(7): 1807-1821.

[9] Tan X, Triggs B. Enhanced local texture feature sets for face recognition under difficult lighting conditions. IEEE transactions on image processing, 2010, 19(6): 1635-1650.

[10] Jobson D J, Rahman Z, Woodell G A. A multiscale retinex for bridging the gap between color images and the human observation of scenes. IEEE Transactions on Image processing, 1997, 6(7): 965-976.

[11] Chen W, Er M J, Wu S. Illumination compensation and normalization for robust face recognition using discrete cosine transform in logarithm domain. IEEE Transactions on Systems, Man, and Cybernetics, Part B (Cybernetics), 2006, 36(2): 458-466.

[12] Wang H, Li S Z, Wang Y. Face recognition under varying lighting conditions using self quotient image IEEE International Conference on Automatic Face and Gesture Recognition, 2004: 819-824.

[13] Chen T, Yin W, Zhou X S, et al. Total variation models for variable lighting face recognition. IEEE transactions on pattern analysis and machine intelligence, 2006, 28(9): 1519-1524. 
[14] Li Q, Yin W, Deng Z. Image-based face illumination transferring using logarithmic total variation models. The visual computer, 2010, 26(1): 41-49.

[15] Xie S, Shan S, Chen X, et al. Fusing local patterns of gabor magnitude and phase for face recognition. IEEE transactions on image processing, 2010, 19(5): 1349-1361.

[16] Hu H. Illumination invariant face recognition based on dual-tree complex wavelet transform. IET Computer Vision, 2014, 9(2): 163-173.

[17] Baradarani A, Wu Q M J, Ahmadi M. An efficient illumination invariant face recognition framework via illumination enhancement and DD-DTCWT filtering. Pattern Recognition, 2013, 46(1): 57-72.

[18] Cheng Y, Hou Y, Zhao C, et al. Robust face recognition based on illumination invariant in nonsubsampled contourlet transform domain. Neurocomputing, 2010, 73(10): 2217-2224.

[19] Zhou Y, Zhou S T, Zhong Z Y, et al. A de-illumination scheme for face recognition based on fast decomposition and detail feature fusion. Optics express, 2013, 21(9): 11294-11308.

[20] Nikan S, Ahmadi M. Local gradient-based illumination invariant face recognition using local phase quantisation and multi-resolution local binary pattern fusion. IET image processing, 2014, 9(1): 12-21.

[21] Wu Y, Jiang Y, Zhou Y, et al. Generalized Weber-face for illumination-robust face recognition. Neurocomputing, 2014, 136: 262-267.

[22] Cheng Y, Li Z, Han Y. A novel illumination estimation for face recognition under complex illumination conditions. IEICE Transactions on Information and Systems, 2017, 100(4): 923-926.

[23] Cheng Y, Jiao L, Cao X, et al. Illumination-insensitive features for face recognition. The Visual Computer, 2017: 1-11.

[24] Tsiotsios C, Petrou M. On the choice of the parameters for anisotropic diffusion in image processing. Pattern recognition, 2013, 46(5): 1369-1381.

[25] Xiao JS, Shan DD, Duan PF, et al. A Fast Image Enhancement Algorithm Based on Fusion of Different Color Spaces. ACTA AUTOMATICA SINICA, 2014, 40(4): 697-705.

[26] Zhu Y, Huang C. An adaptive histogram equalization algorithm on the image gray level mapping. Phys Procedia , 2012, 25: 601-608.

[27]K.C. Lee. The Extended Yale Face Database B , http://vision.ucsd.edu/ iskwak/ExtYaleDatabase/ExtYaleB.html

[28] Hu H. Illumination invariant face recognition based on dual-tree complex wavelet transform. IET Computer Vision, 2014, 9(2): 163-173.

[29]T. Sim, S. Baker, and M. Bsat. The CMU pose, illumination, and expression database, IEEE Trans. Pattern Anal. Mach. Intell, 2003,25: 1615-1618.

[30] W. Gao, B. Cao, S. Shan, D. Zhou, X. Zhang, and D. Zhao. CAS-PEAL Face Database, http://www.jdl.ac.cn/peal/index.html.

[31] Ding J, Wen C, Li G, et al. Locality sensitive batch feature extraction for high-dimensional data. Neurocomputing, 2016, 171: 664-672.

[32] Belhumeur, P. N., Hespanha, J. P., \& Kriegman, D. J .. Eigenfaces vs. fisherfaces: Recognition using class specific linear projection. IEEE Transactions on pattern analysis and machine intelligence, 1997, 19(7):711-720.

[33] Liu C. Capitalize on dimensionality increasing techniques for improving face recognition grand challenge performance. IEEE transactions on pattern analysis and machine intelligence, 2006, 28(5): 725-737.

[34] Martínez A M, Kak A C. Pca versus lda. IEEE transactions on pattern analysis and machine intelligence, 2001, 23(2): 228-233. 